\title{
J-Integral Analysis: An EDXD and DIC Comparative Study for a Fatigue Crack
}

\author{
A. Koko ${ }^{a^{*}}$, P. Earpa ${ }^{\mathrm{a}}$, T. Wigger ${ }^{\mathrm{b}}$, J. Tong ${ }^{\mathrm{b}}$, T.J. Marrow ${ }^{\mathrm{a}}$ \\ a Department of Materials, University of Oxford, Oxford OX1 3PH, UK \\ b School of Engineering, University of Portsmouth, Anglesea Building, Anglesea Road, \\ Portsmouth PO1 3DJ, UK \\ * Corresponding author. \\ E-mail address: abd.mohamed@materials.ox.ac.uk
}

\section{Abstract}

Synchrotron Energy Dispersive X-ray Diffraction (EDXD) and Digital Image Correlation (DIC) have been applied to map, simultaneously, the $2 \mathrm{D}$ elastic strain and displacement fields of a propagating fatigue crack in the HAZ of a welded Cr2Ni4MoV bainitic steel. The position of the crack tip was tracked via a phase congruency analysis of the displacement field, and also by detection of its cyclic plastic zone. Both types of full field data provided independent inputs to finite element/J-integral analyses that directly quantified the elastic cyclic stress intensity factor range applied to the crack. No knowledge was required of the specimen geometry, crack length or applied loads. The agreement between the two analyses in this controlled study shows that strain mapping by synchrotron EDXD can provide a reliable method to study the crack fields in more complex problems, such as interactions between crack closure, residual stresses and applied loading.

Keywords:

EDXD, DIC, Crack tip, J-integral, finite element analysis, Fatigue crack growth 


\section{Introduction}

Under small scale yielding (SSY) conditions, fatigue crack propagation is thought to be dictated by the surrounding elastic strain field, albeit mediated by the limited plasticity that may occur around the crack tip. The elastic strain field around a crack can be described by the elastic strain energy release rate, or stress intensity factor. Fatigue crack propagation rates are generally related to the range of the stress intensity factor, which describes the combined effects of crack size, applied loads and the geometry of a cracked body on the fatigue crack growth behaviour.

Cherepanov [1] and Rice [2] showed that the Riemann-summation of the non-linear energy release rate can be written using Green's theorem (Equation 1) as a path-independent contour integral (known as the J-integral, Figure 1) around a crack tip that exhibits the Hutchinson[3], Rice and Rosengren [4] (HRR) singularity. Here, $J$ is the J-integral, $\sigma_{i j}$ and $\epsilon_{i j}$ are the stress and strain tensors, respectively, $W$ is the strain energy density, $T_{i}$ are the components of the traction vector, $u_{i}$ the displacement vector components, $n_{j}$ the components of the unit vector normal to $\Gamma$, and $d s$ is the length increment along the contour.

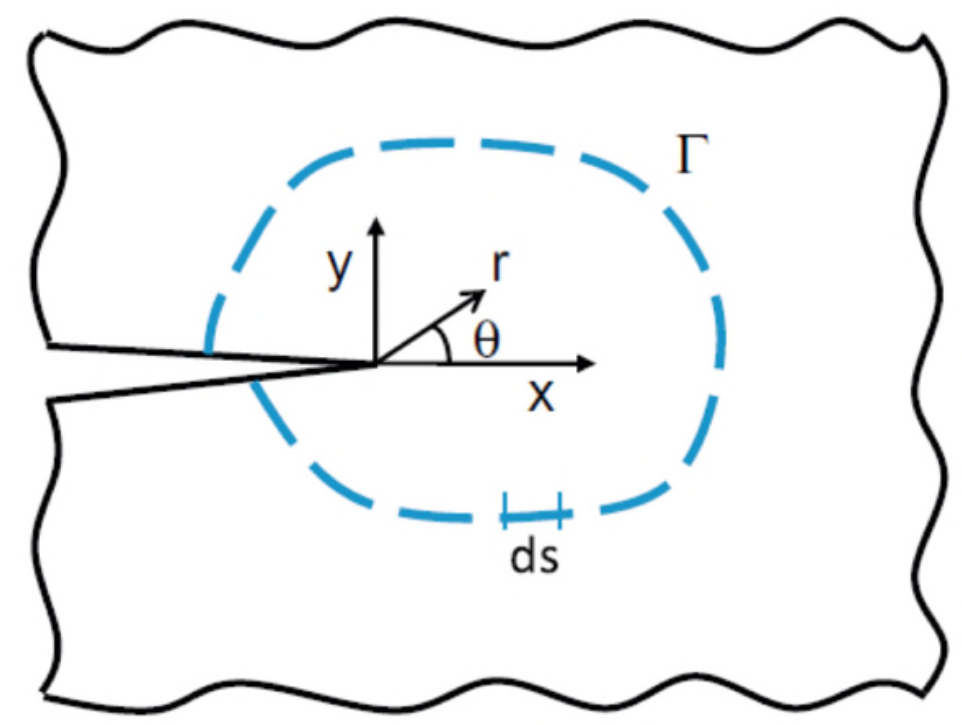

Figure 1: An arbitrary contour $(\Gamma)$ for the line integral evaluation of the J-integral [5]. 


$$
J=\int_{\Gamma}(W d y-\underbrace{T_{i} \frac{\partial u_{i}}{\partial x} d s}_{\text {Dissipated energy }}), W=\int_{0}^{\varepsilon_{i j}} \sigma_{i j} d \epsilon_{i j}, T_{i}=\sigma_{i j} n_{j}
$$

The elastic strain field acting on the crack tip can therefore be described using the J-integral. The critical J-integral $\left(U_{I C}\right)$ - at the point of crack propagation - can then be used as a fracture criterion [6], so long as the J-integral is independent of specimen geometry and load condition. Similarly, the range of J-integral within cycles of fatigue loading can be related to rates of fatigue crack propagation [7], [8]. The integral nature of $J$ facilitates its use in finite element (FE) analysis, as a line, surface (2D) or volume (3D) integral, and also the decomposition of modes of loading [9]. Thus, in experimental studies and structural integrity assessments, it is common to calculate the J-integral by FE methods from the knowledge of the applied boundary conditions (loads) and the specimen geometry. To verify the validity of such analyses, and to critically assess their ability to account for plasticity and residual stresses [10], it is of interest to explore the experimental methods that would allow direct assessment of the J-integral.

Direct evaluation of the J-integral from a measured crack displacement field using a finite element method was first demonstrated in the JMAN method [11]. This takes as its input the full-field displacements from an image correlation analysis and the J-integral is then calculated using the element-based virtual crack extension formulation [12]. A later development was the JMAN-S method [13], which used the elastic strain field measured via diffraction. This is first solved to obtain the equivalent displacement field, from which the Jintegral is calculated. The JMAN-S method, which makes no assumptions of the form of the crack field, has been applied to investigate crack fields in materials as diverse as bainitic steel [13], polygranular graphite with non-linear properties [14] and polycrystalline ferro-electric materials [15].

Thus, in this study, the surface displacement field from DIC measurement and the bulk elastic strain field probed within the specimen using EDXD have been compared for the first time in a study of a fatigue crack in the heat affected zone of a bainitic steel. The primary objective of this work is to demonstrate that full field mapping using EDXD provides a reliable measure of the elastic field within the test specimen, from which the effects of cyclic loading may be 
directly measured without requiring knowledge of the specimen geometry, applied loading or residual fields. This may then provide an experimental tool for future studies that would explore interactions between applied loading and internal fields, such as crack closure or residual stresses from overload cycles, or to assess the cyclic fields applied to cracks within bulk components under complex loading.

\section{Experiment}

Full details of the experiment that provided the raw data analysed in this work have been published elsewhere [16], and are summarised briefly here. The test specimen was fabricated from a HSLA ('high strength low alloy', yield strength 800 MPa) steel weld joint (Cr2Ni4MoV), which had been annealed at $550^{\circ} \mathrm{C}$ for 40 hours to relieve the welding residual stresses. A standard compact tension geometry (notch depth of $a / W=0.35, W=50 \mathrm{~mm}$ ) was used, with a thickness of $6.5 \mathrm{~mm}$ to improve X-ray transmission (Figure 2). The specimen, which satisfied the ASTM E647 conditions for elastic deformation, was fatigued via a load-shedding scheme to a final $\Delta K$ of $13 \mathrm{MPa} V \mathrm{~m}(R=0.1)$ to develop a pre-crack in the $\mathrm{HAZ}$, parallel to the weld interface as shown in Figure 2.

One surface of the specimen was metallographically polished, and a region of interest $(10 \times$ $10 \mathrm{~mm}$ ) around the crack tip was etched ( $3 \%$ nitric acid-ethanol solution) to provide sufficient speckle for digital image correlation of optical images. These were recorded during the experiment using a LaVision DIC system (LaVision GmbH, Göttingen, Germany), equipped with a 12 bit CCD camera attached to an optical microscope with a Schneider Kreuznach $50 \mathrm{~mm}$ lens and a $100 \mathrm{~mm}$ extension tube. The distance between the lens and specimen surface was approximately $50 \mathrm{~mm}$. Two LED flashlights were used to ensure appropriate lighting conditions. An area around the crack tip of approximately $1300 \mu \mathrm{m} \times 1100 \mu \mathrm{m}$ was imaged with a spatial resolution of $0.54 \mu \mathrm{m} /$ pixel.

The Energy Dispersive X-ray Diffraction measurements (EDXD) were obtained using a multiwavelength "white beam" and the 'Horseshoe' 23-element detector (Figure 4.A) of beamline I12 (Joint Engineering, Environmental and Processing - JEEP) at the UK Diamond Light Source [17]. This provided the diffraction spectra from suitably oriented grains within a gauge 
volume defined by collimating slits and diffraction angle, which in this case was $100 \mu \mathrm{m} \times 100$ $\mu \mathrm{m} \times 4500 \mu \mathrm{m}$. Rastering of the specimen position in the beam allowed mapping of an area of $1.6 \mathrm{~mm} \times 1.3 \mathrm{~mm}$ ( 208 data points per map) around the crack tip.

Optical and EDXD data were obtained pseudo-simultaneously by installing the EDXD detector and the DIC microscope next to each other. The specimen was loaded by a $100 \mathrm{kN}$ servohydraulic loading rig that was mounted on a translation stage, so the specimen could be positioned alternately in the X-ray beam for EDXD measurements and then in the view of the microscope for optical imaging. The time required to change between optical and EDXD modes was less than 10 minutes. The translation stage positioning was carried out using a calibrated digital encoder, with a precision greater than $\pm 10 \mu \mathrm{m}$.

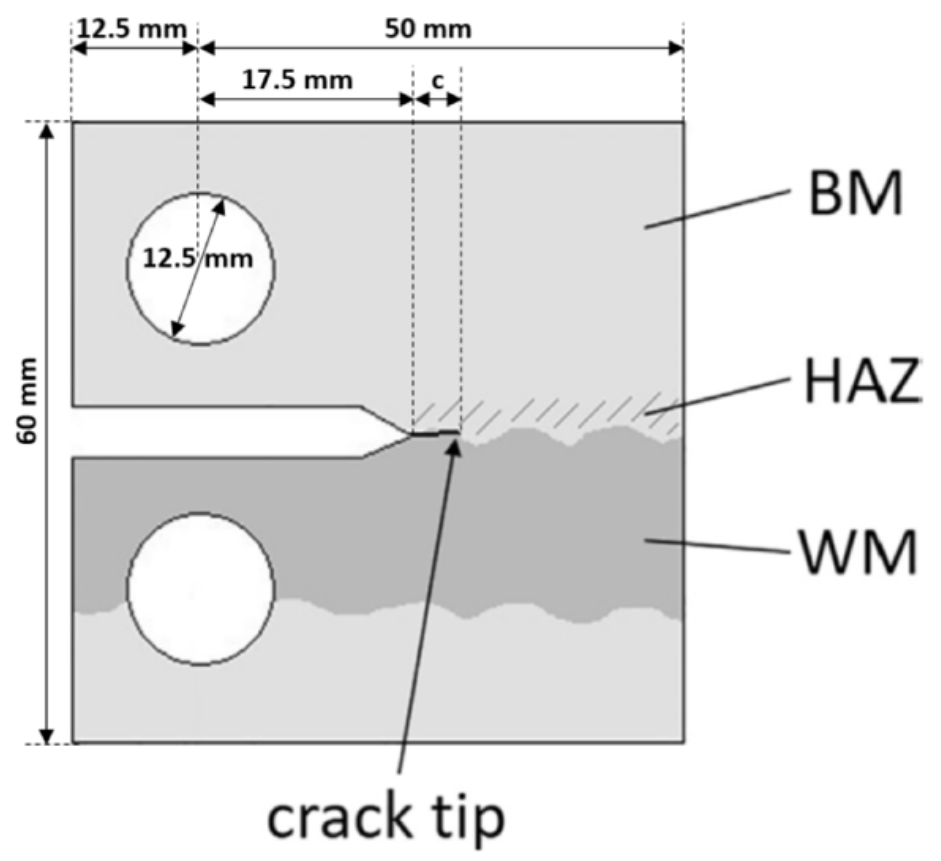

Figure 2: Schematic of the compact tension (CT) specimen, identifying the weld metal (WM), heat affected zone (HAZ), base metal (BM) and the fatigue pre-crack location in the HAZ.

The experiment was executed on a single test specimen in 3 phases. These are summarised in Figure 3, which presents the applied stress intensity factor as a function of the number of load cycles applied, where K:ASTM was calculated from the specimen geometry, measured crack length and applied load using the ASTM standard E647 [18] . Here, $P$ is the applied load, $B$ the sample thickness, $a$ the crack length and $W$ the sample width. 


$$
K_{I}=\frac{P}{B} \sqrt{\frac{\pi}{W}}\left[\begin{array}{c}
16.7\left(\frac{a}{W}\right)^{\frac{1}{2}}-104.7\left(\frac{a}{W}\right)^{\frac{3}{2}}+369.9\left(\frac{a}{W}\right)^{\frac{5}{2}} \\
-573.8\left(\frac{a}{W}\right)^{\frac{7}{2}}+360.5\left(\frac{a}{W}\right)^{\frac{9}{2}}
\end{array}\right]
$$

In the $1^{\text {st }}$ phase, the specimen was cycled between upper $\left(P_{\max }\right)$ and lower $\left(P_{\min }\right)$ load values of $3.4 \mathrm{kN}$ and $0.34 \mathrm{kN}$ respectively (stress intensity factor range $K_{\max }-K_{\min }=\Delta K$, where $\Delta K \approx 15 \mathrm{MPa} V \mathrm{~m}, R=0.1$ ) to observe the evolution of the crack field without substantial fatigue crack growth. Observations were obtained at intervals with the specimen under a static load; initially at $P_{\min }$, and then at $P_{\max }$ for up to 1000 cycles in total. In the $2^{\text {nd }}$ phase, the loads were increased to $6.6 \mathrm{kN}$ and $0.66 \mathrm{kN}$ values ( $P_{\max }$ and $P_{\min }$ respectively) to provide a stress intensity factor range of $\Delta K \approx 30 \mathrm{MPa} \vee \mathrm{m}, R=0.1$ to examine the crack field developed during steady-state fatigue crack propagation. Measurements were obtained initially at $P_{\min }$, and then at $P_{\max }$ for up to 2000 cycles in total. In the $3^{\text {rd }}$ phase, a single load cycle between $0.66 \mathrm{kN}$ and $3.3 \mathrm{kN}$ was examined as the load was incrementally increased and then decreased without any crack growth in order to examine the variation of the crack field within a single load cycle.

After the experiment, the specimen was sectioned metallographically and electron backscatter diffraction (EBSD) was used to investigate the microstructure surrounding the crack tip. EBSD maps at a step size of $43 \mathrm{~nm}$, over an area of $0.5 \mathrm{~mm} \times 0.5 \mathrm{~mm}$, were obtained using a ZEISS Merlin FEG SEM microscope fitted with a Bruker EBSD system and plasma cleaner. The data was analysed using MTEX [19], and texture was calculated as an Orientation Distribution Function (ODF) with a halfwidth of $7.5^{\circ}$ to minimise the estimation error to less than $0.1 \%[20]$. 


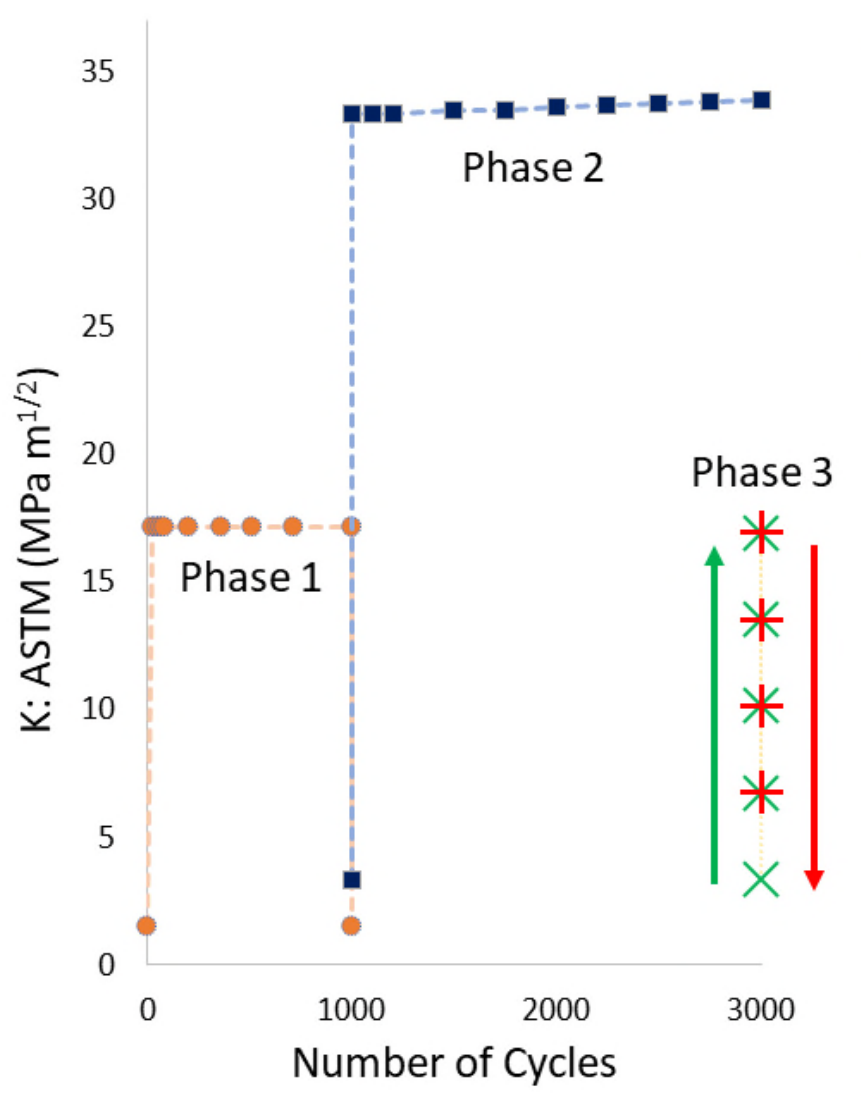

- Cycling at 3.4kN/0.34kN (Pmax/Pmin)

- Cycling at $6.6 \mathrm{kN} / 0.66 \mathrm{kN}$ (Pmax/Pmin)

$\times$ Single Cycle (up)

+ Single Cycle (down)

\section{Phase 3}

Figure 3: The phases of the experiment, identifying the applied stress intensity factor, K: ASTM (calculated from the crack length, specimen geometry and loads using the ASTM standard formula), for each observation as a function of the total number of fatigue cycles.

\section{Data Analysis}

A typical X-ray spectra from one element of the detector is illustrated in Figure 4a, which shows the diffracted intensity as a function of $q$, where $q=2 \pi / d$ and $d$ is the diffracting lattice plane spacing. The most intense peak is from the $\{110\}$ planes; an example of a single peak fit for the $\{110\}$ peak with its FWHM (Full Width Half Maximum) intensity identified is shown as an inset in Figure 4a. To correct for different sensitivities of the elements in the detector, calibration data were obtained using an untextured Ceria powder $\left(\mathrm{Ce}_{2} \mathrm{O}\right)$ sample using the same parameters (e.g. beam slits and collimation), and were used to normalise ${ }^{1}$ the measured intensity for each of the 23 elements (see inset Figure $4 a$ ) with respect to one

\footnotetext{
${ }^{1}$ The MATLAB code is available at https://bit.ly/33Uwdz9.
} 
element, arbitrarily selected as number 12 at $90^{\circ}$. Normalised data from the full set of 23 elements are shown in Figure 4b.

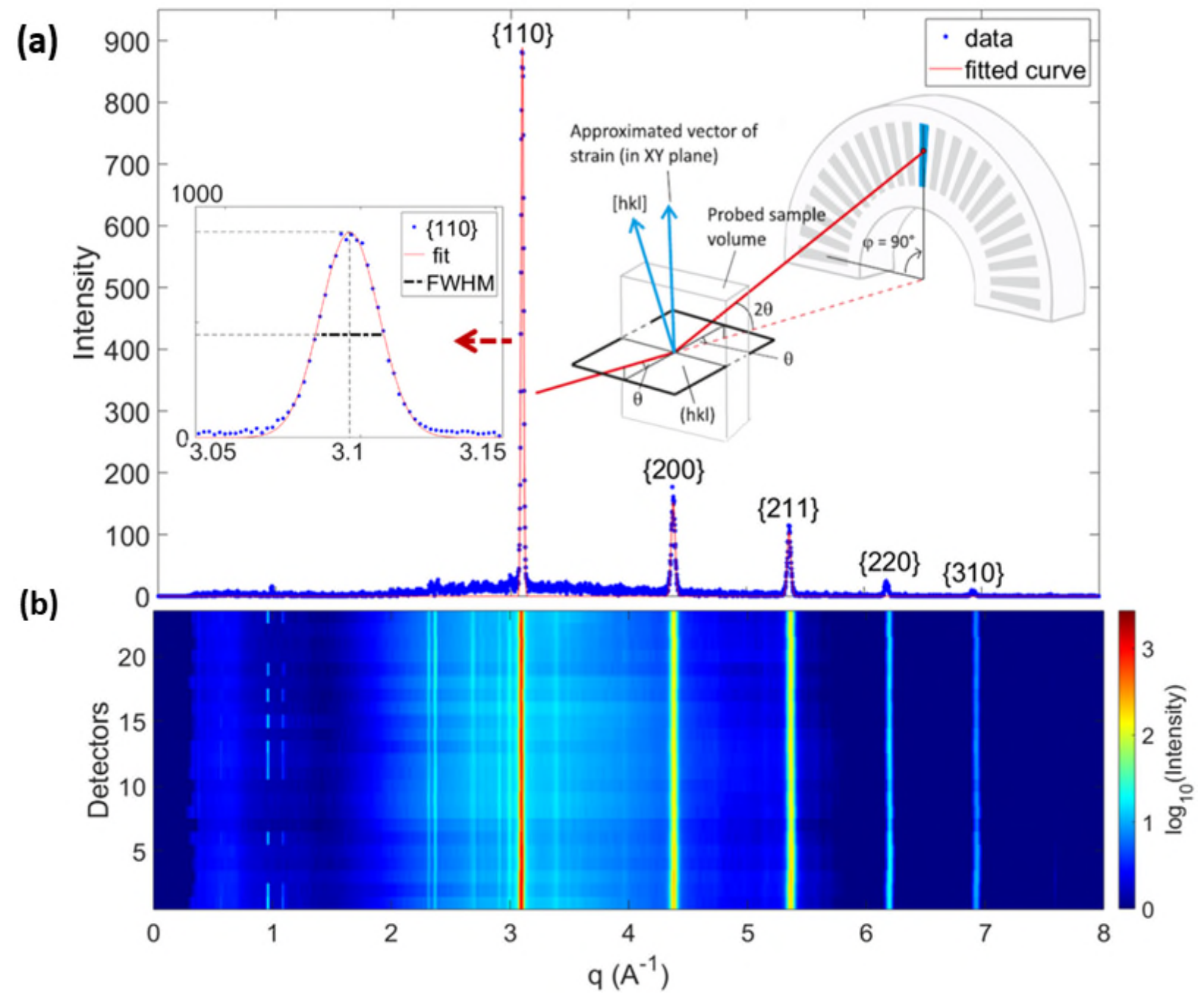

Figure 4: (a) Energy dispersive X-ray diffraction (EDXD) spectrum obtained at 1 point from a single element of the 'Horseshoe' detectors; a Gaussian fit to extract peak position and FWHM from one detector is shown as an inset. (b) normalised data from all 23 elements of detector.

The EDXD spectra were fitted to obtain the peak positions $\left(q_{n}^{h k l}\right)$ from which to calculate the strain $\left(\epsilon_{n}^{h k l}\right)$ on each family of lattice planes relative to an unstrained reference value, $q_{0}$, using equation (3). In a development of the method of Simpson [21] and Mostafavi [22], the overall lattice strain $\left(\epsilon_{\varphi}^{h k l}\right)$, as a function of azimuthal angle $(\varphi)$ at each detector was weighted $\left(W_{n}^{h k l}\right)$ for the contribution of each $(n)$ diffraction peak, relative to all peaks $(N)$ as described in equations (4) and (5), using a 'relative' peak intensity $\left(I_{n}^{h k l}\right)$ that depends on the multiplicity of the diffracting planes [23]. The elastic strain variation across the detectors was then fitted 
using a single-term Fourier transform, and the components of the elastic strain tensor $\left(\epsilon_{x x}\right.$, $\epsilon_{x y}$ and $\epsilon_{y y}$ ) were extracted from the fitting parameters using equation (6) (Figure 5a).

$$
\begin{gathered}
\epsilon_{n}^{h k l}=\frac{q_{0}}{q_{n}^{h k l}-1} \\
\epsilon_{\varphi}^{h k l}=\sum_{n=1}^{N} \epsilon_{n}^{h k l} W_{n}^{h k l} \\
W_{n}^{h k l}=I_{n}^{h k l} / \sum_{n=1}^{N} I_{n}^{h k l} \\
\epsilon_{\varphi}^{h k l}(\varphi)=\left[\frac{\epsilon_{x x}+\epsilon_{y y}}{2}\right]+\left[\frac{\epsilon_{x x}-\epsilon_{y y}}{2}\right] \cos 2 \varphi+\frac{\epsilon_{x y}}{2} \sin 2 \varphi
\end{gathered}
$$

The Full-Width Half Maxima $\left(F W H M_{\varphi}^{h k l}\right)$ were calculated using the Gaussian-fit peak width $\left(c_{n}^{h k l}\right)$ as in equation (7). The mean value across all the detectors was used to map $\mathrm{FWHM}_{\mathrm{xy}}$ at each $(x, y)$ coordinate measurement point. The maximum value of FWHM was quite localised (Figure 5b), and a cross-correlation algorithm could be used for efficient subpixel image registration between the FMHM maps [16]. This allowed changes in the position of the FWHM peak (or maximum spatial value) to be tracked with subpixel accuracy ( 1 pixel $=100$ $\mu \mathrm{m})$.

$$
F W H M_{\varphi}^{h k l}=\sum_{n=1}^{N} 2 \sqrt{2 \ln (2)} c_{n}^{h k l} W_{n}^{h k l}
$$


(a)

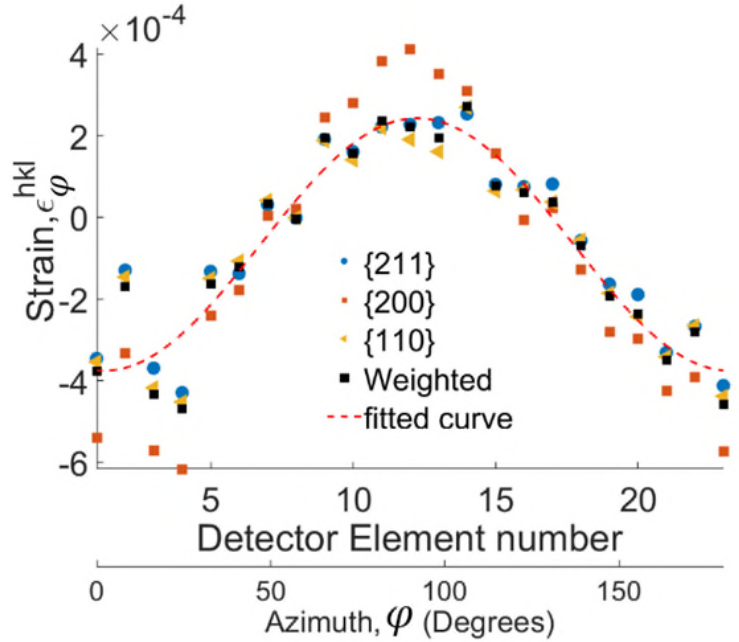

(b)

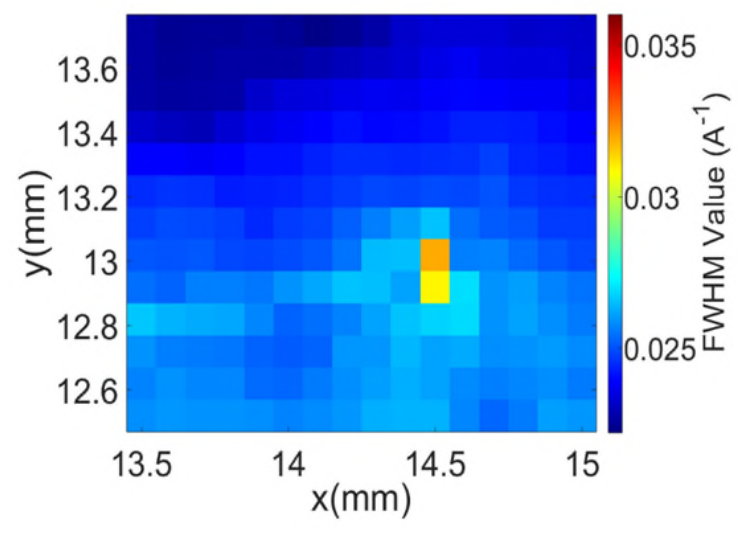

Figure 5: a) the variation of elastic strain with azimuth angle over the 23 detectors for the dominant single peaks, their weighted average and the fitted curve to the weighted average; $b$ ) an example map of weighted FWHM (full width half maximum) value. The crack tip position can be tracked by a crosscorrelation analysis of the movement of the distribution of FWHM values between successive maps.

Maps of the effect of the change in load (i.e. from $P_{\min }$ to $P_{\max }$ ) on the strain tensor components were obtained by subtracting, at each measurement point, the apparent strains measured in the preceding observation of the specimen (i.e. at $P_{\min }$ ). A set of typical strain maps is shown in Figure 6. The reference maps at $P_{\min }$ are shown in Figure 6 (I-III); the crosses show the positions of the individual measurements with interpolated contours of strain. The strain changes from $P_{\min }$ to $P_{\max }$ are shown in Figure 6 (IV-VI). Here, due to slight stage movements during the observations, the measurement window is not completely the same and some small regions at the margin do not have measurements at both $P_{\min }$ and $P_{\max }$. These are shown as white.

Normally, XRD data from un-strained region of the sample would be used for the reference, with reference peak positions $\left(q_{o}\right)$ obtained by Gaussian fitting to the most intense - also distinct - peaks (i.e. $\{110\},\{200\}$ and $\{211\}$ in body centred cubic ferrite) to minimise fitting residuals and error. In this experiment, a satisfactory un-strained reference could not be obtained; the specimen is from a weld and prior to the experiment there are no locations that can be assumed to be stress free, even in the unloaded state and after a stress-relief heat treatment. Hence an alternative approach was required to find a suitable reference with minimum strain. 
First, theoretical positions for the most intense peaks were calculated with a nominal lattice parameter ( $a_{o}=2.8665 \AA$ for ferrite). Then, a set of measurement points remote from the crack tip (examples indicated in Figure 6. III) was identified which had minimum absolute strains (i.e. minimum elastic deformation) and minimum FWHM (i.e. minimum plastic deformation). These points were averaged to provide a more representative reference for $q_{o}$, as shown in Table 1, which was used to recalculate the elastic strain tensor components in that phase of the experiment (the sample was removed and replaced in the loading rig between phases 2 and 3).

Table 1: Optimised $q_{0}$ values, used for the reference in strain maps, for the most strongly diffracting planes. All measured at $P_{\min }$ (Phase I and Phase 3: $0.33 \mathrm{kN}$, Phase 2: $0.66 \mathrm{kN}$ ).

\begin{tabular}{|c|c|c|c|c|}
\hline \multirow{2}{*}{ Planes } & \multirow{2}{*}{$\begin{array}{l}q_{0}^{\text {Theoretical }} \\
\left(\AA^{-1}\right)\end{array}$} & \multicolumn{3}{|c|}{$q_{0}^{\text {Optimised }}\left(\AA^{-1}\right)$} \\
\hline & & $1^{\text {st }}$ Phase & $2^{\text {nd }}$ Phase & $3^{\text {rd }}$ Phase \\
\hline$\{110\}$ & 3.10041 & $3.09560 \pm 8.8^{*} 10^{-6}$ & $3.09633 \pm 1.1 * 10^{-5}$ & $3.09570 \pm 9.5 * 10^{-6}$ \\
\hline$\{200\}$ & 4.38464 & $4.37774 \pm 2.4 * 10^{-5}$ & $4.37872 \pm 2 * 10^{-5}$ & $4.37792 \pm 2.1 * 10^{-5}$ \\
\hline$\{211\}$ & 5.37006 & $5.36173 \pm 2.8 * 10^{-5}$ & $5.3630 \pm 2 * 10^{-4}$ & $5.36202 \pm 2.3 * 10^{-5}$ \\
\hline
\end{tabular}

\pm in optimised $q_{0}$ values reflect the standard deviation from the mean in the selected points. 

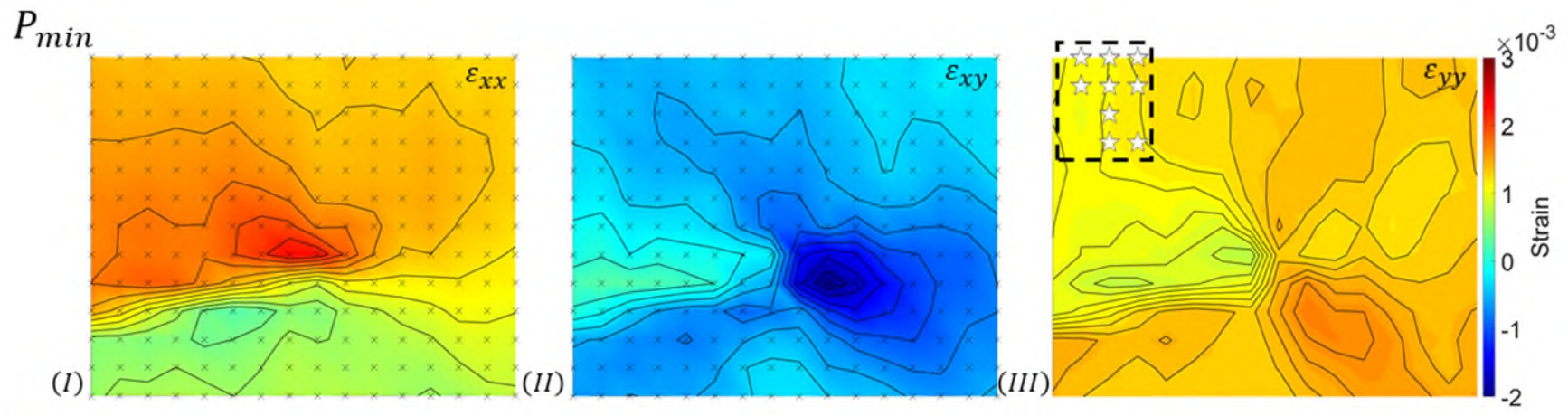

$P_{\max }-P_{\min }$
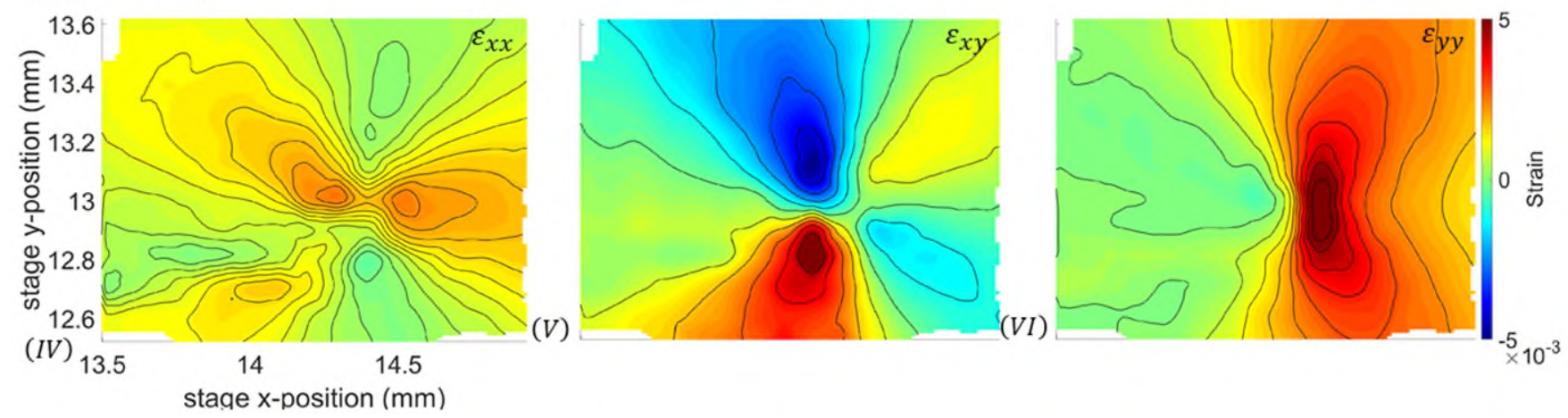

Figure 6: Examples of strain tensor maps obtained at (I-III) $0.66 \mathrm{kN}\left(P_{\min }\right)$ and (IV-VI) $6.6 \mathrm{kN}\left(P_{\max }\right)$ for $\varepsilon_{\mathrm{xx}}$ $\varepsilon_{\mathrm{xy}}$ and $\varepsilon_{\mathrm{yy}}$. The strains at $P_{\min }$ are calculated using the theoretical $q_{0}$ (see Table 1). The points selected for the optimised $q_{0}$ are indicated by the white stars inside the dashed black box in (III).

The optical images allowed use of digital image correlation (DIC) to measure the displacement field between a reference image and a deformed image [24]. Some repositioning of the lighting was necessary between observations, and despite the precision of the translation stage the optical images suffered from translational rigid body movements and changes in light intensity. Image registration to improve the accuracy of subsequent DIC analysis was implemented via an efficient Fourier-space subpixel image registration MATLAB ${ }^{2}$ algorithm using cross-correlation to calculate shifts [25]. The corrected data were processed using the DaVis Software (FFT DIC algorithm) using a subset size of $48 \times 48$ image pixels with $25 \%$ overlap to obtain the displacement field.

The crack position and crack tip position were identified using the displacement field by a method developed by Cinar et al. [26], which uses the phase congruency [27]. The sequence of the data post-processing is summarised in Figure 7 where a phase congruency map of the

\footnotetext{
${ }^{2}$ xCorrRBMCorrection code is available at https://bit.ly/31YVMgA.
} 
smoothed and filtered displacement field is computed. A Hough transform is applied to detect (i.e. binarise) the linear features; the longest linear discontinuity (i.e. the crack) is then identified autonomously.

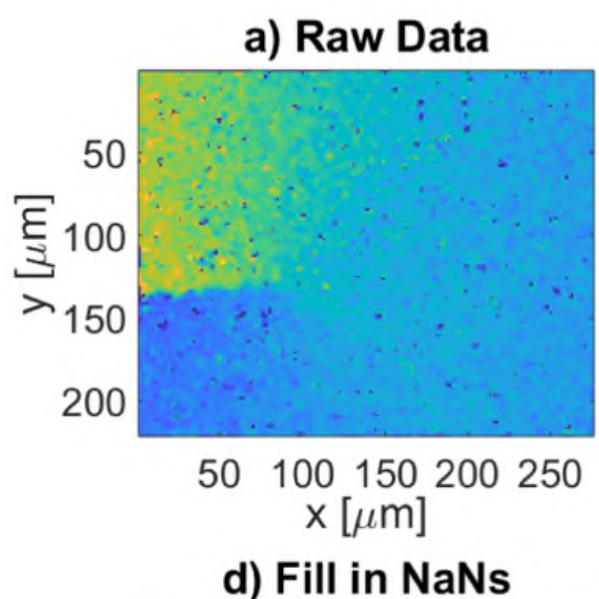

b) Fill in NaNs

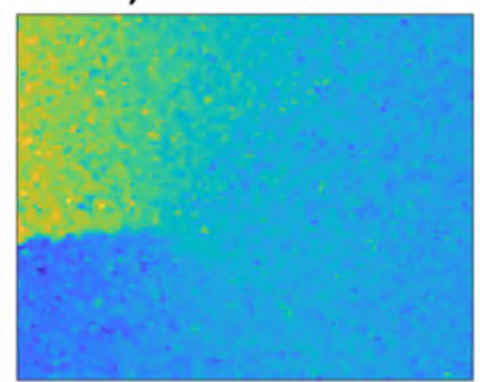

e) Phase Congruency

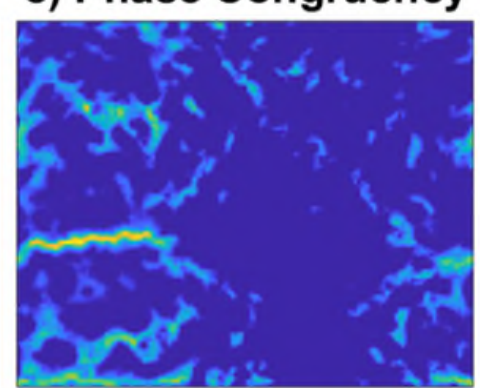

c) Median Filtering

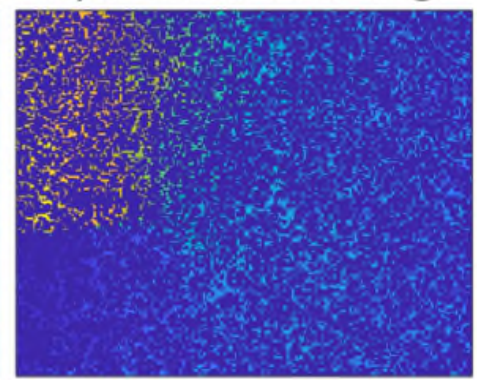

f) Binarize the Image

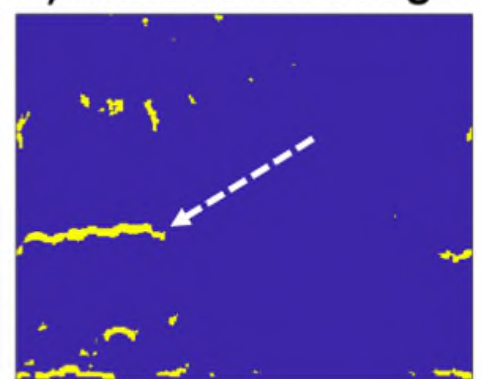

Figure 7: The post-processing steps of the phase congruency analysis [26]: to identify the crack from the DIC-measured displacement field: a) raw data ( $y$-displacement field); b) interpolation to fill empty points with no DIC data for to insufficient correlation coefficient ( $N a N$ : 'not a number'); $c$ ) median filter to reduce noise; d) final interpolation to remove any remaining points with no data; e) calculation of phase congruency values; f) binary segmentation and identification of the longest linear feature using Hough transform .

\section{Results and Discussion}

The developed fatigue crack, imaged by SEM, is shown in Figure 8a, and an EBSD grain orientation map of the same area is presented in Figure 8b. The pole figures obtained from this map (Figure 8c) shows a weak texture. This is consistent with the EDXD data (Figure 4b), which showed negligible variation between detectors after the Ceria normalisation for sensitivity. These data were used with the ISODEC software [28] to calculate the diffraction elastic constants in the direction normal to the crack plane, assuming $E_{110}=220 \pm 2 \mathrm{GPa}$, 
$E_{200}=164 \pm 2 \mathrm{GPa}$ and $E_{310}=175 \pm 1 \mathrm{GPa}$ [29]: a value of $212 \pm 7 \mathrm{GPa}$ was obtained for Young's modulus and 0.318 for Poisson's ratio using Hill's weighted average model [30]. The EBSD data also allowed mapping of the lower bound estimate of the number density of geometrically necessary dislocations (GND) [31]; the increased GND density along the crack path is apparent (Figure 8d).

(a)

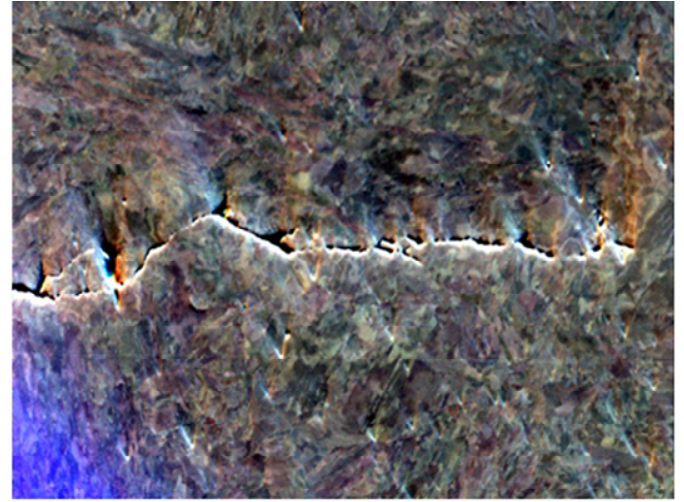

(c)

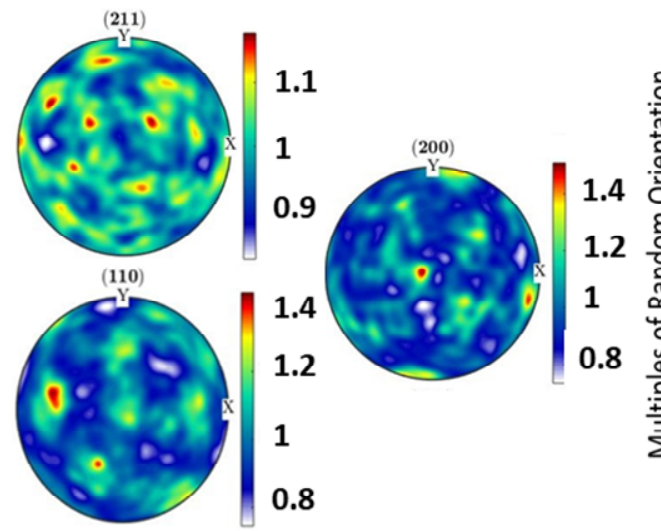

(b)

(d)

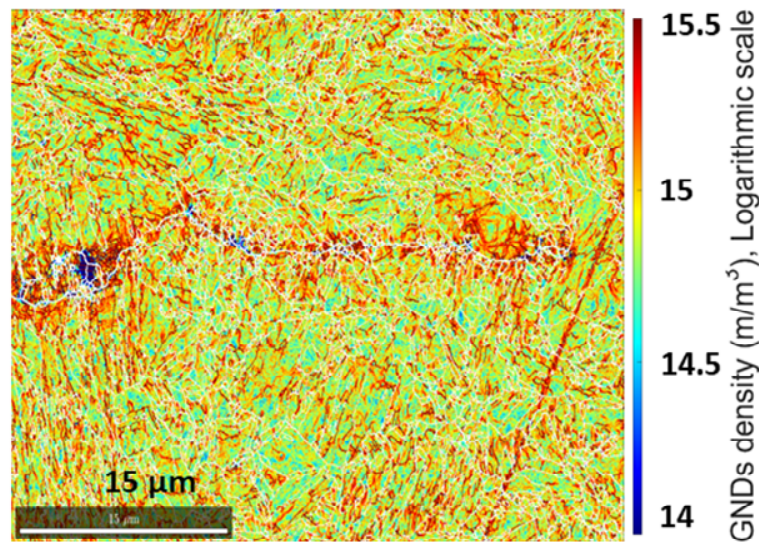

Figure 8: SEM examination of the fatigue crack: (a) secondary electron mage; (b) EBSD map of the same area as (a). (c) Orientation Distribution Function (ODF) plots for the $\{211\},\{200\}$ and $\{110\}$ planes; d) geometrically-necessary dislocations (GNDs) density.

The GND distribution shows the very limited extent of crack tip plasticity of the propagating fatigue crack. The localised increase of diffraction peak width (i.e. the weighted FWHM) can be attributed to the effect of plastic deformation at the crack tip, so the cross-correlation tracking of the position of the FWHM peak (e.g. Figure 5b) can be used to investigate changes in the crack tip position. The results are presented in Figure 9. The change in crack tip position 
identified via the phase congruency analysis of the DIC-measured surface displacement field is also shown.

In phase 1 of the experiment, there was no change of crack position observed by DIC. However, over the first 100 cycles a significant change occurred in the position of the FWHM peak, measured at $3.4 \mathrm{kN} P_{\max }$, before becoming essentially static. This may be related to a gradual evolution of the crack tip plastic zone with the increase in applied maximum stress intensity factor relative to the lower value used to develop the pre-crack. Reduction of the load to $0.3 \mathrm{kN} P_{\min }$ at the end of phase I was accompanied by a retreat of the FWHM peak position, and increasing the load to $0.6 \mathrm{kN}\left(P_{\min }\right.$, phase 2$)$ caused an increase in the position of the FWHM peak.

In phase 2 , the change in position of the FWHM peak, measured at $6.6 \mathrm{kN} P_{\max }$, was generally quite consistent with the change in position of the crack tip measured by DIC. Differences may be due to the shape of the crack front, since the DIC measurement was made at the surface, while the EDXD data are averaged across a thickness of $4.5 \mathrm{~mm}$ of the gauge volume of the diffracted beam [16] that is within the specimen. These data show that observations of the EDXD FWHM peak position at a nominally constant applied stress intensity factor may be used to detect and measure crack propagation. 


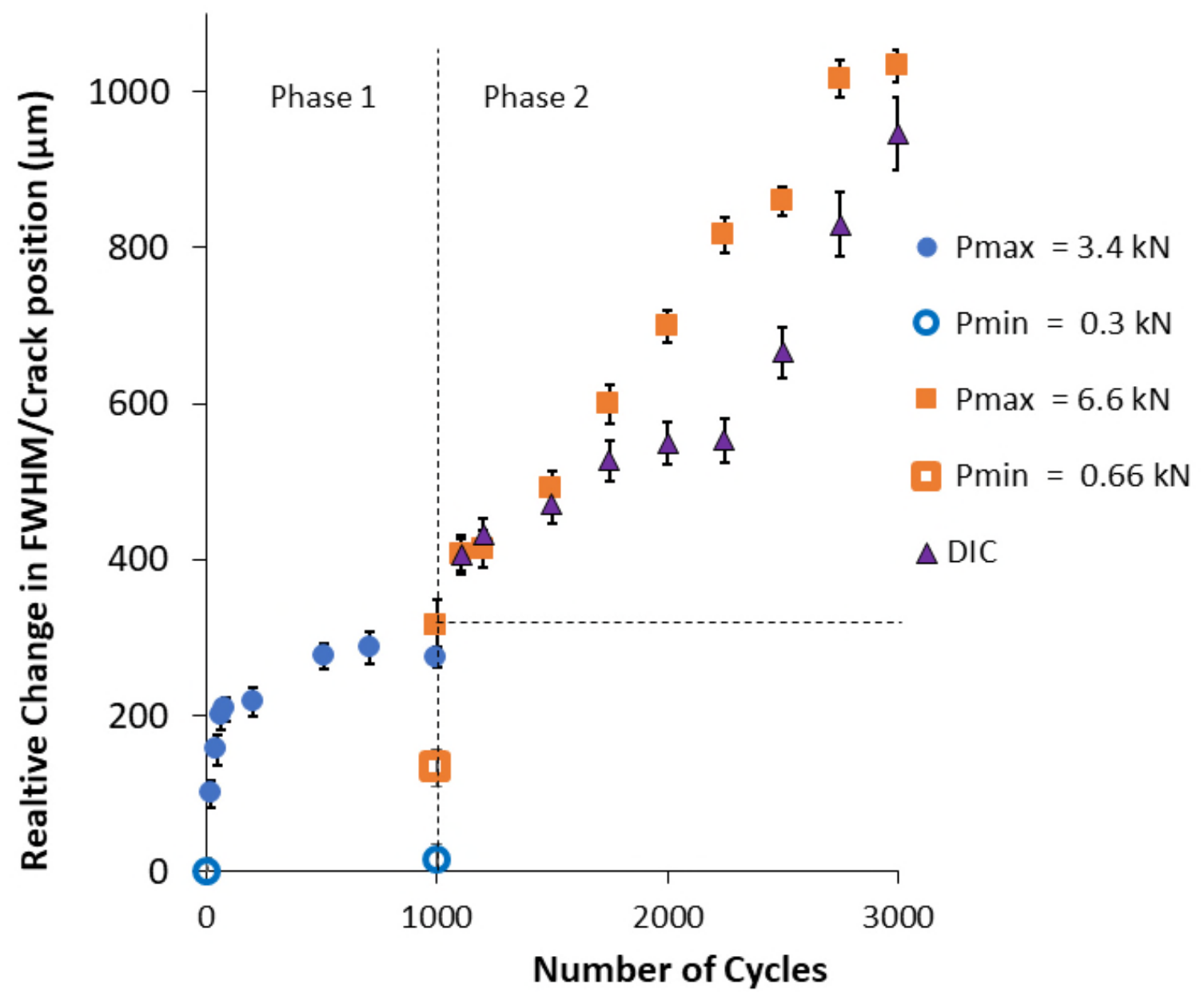

Figure 9: The change in crack tip position with number of cycles, measured using EDXD and DIC. In phase I, there was no detectable change in crack tip position by DIC. In phase 2 , the DIC-measured change has been plotted relative to the position of the EDXD FWHM peak, measured at $P_{\max }$ at the start of phase 2. (i.e. at the intersection of the orthogonal dotted lines)

\section{J-integral analysis}

The EDXD elastic strain tensor maps were used to calculate the J-integral using the JMAN-S method [13], and the displacement fields from DIC were used to calculate the J-integral using the J-MAN method [11]. J-integral evaluation using these methods requires masking of the crack path, and examples of the selection of the initial contour are shown in Figure 10. The outer contour extends to the bounds of the data, and the J-integral analysis uses the converged value with increasing contour size. Both methods are currently limited to mode (I) loading. This was warranted by the sample geometry and loading and is verified by inspection of the displacement field. The calculations, using these 2D fields, assumed plane stress 
conditions for the JMAN analysis (i.e. surface measurements by DIC) and plane strain conditions for JMAN-S (i.e. bulk measurements within the specimen by EDXD). The same ISODEC-calculated elastic properties were used in all analyses. The converged J-integral values were then converted to linear elastic stress intensity factors using the appropriate plane stress and plane strain relationships.

(a)

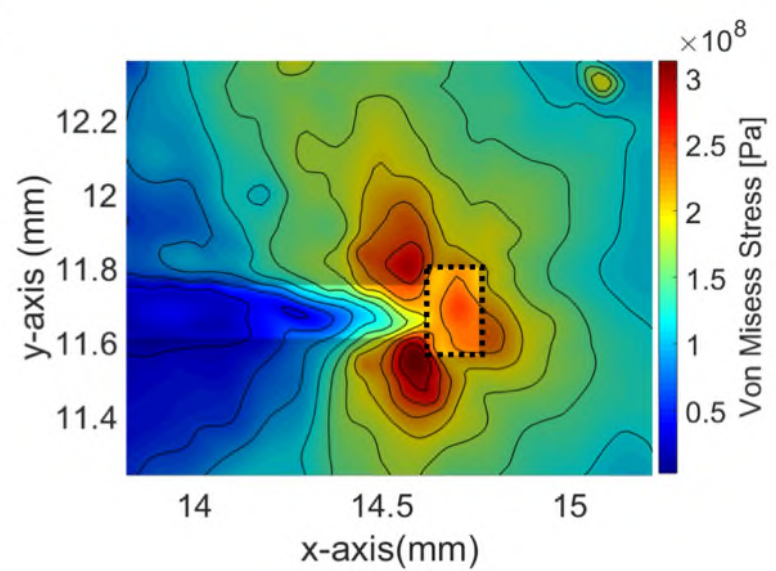

(b)

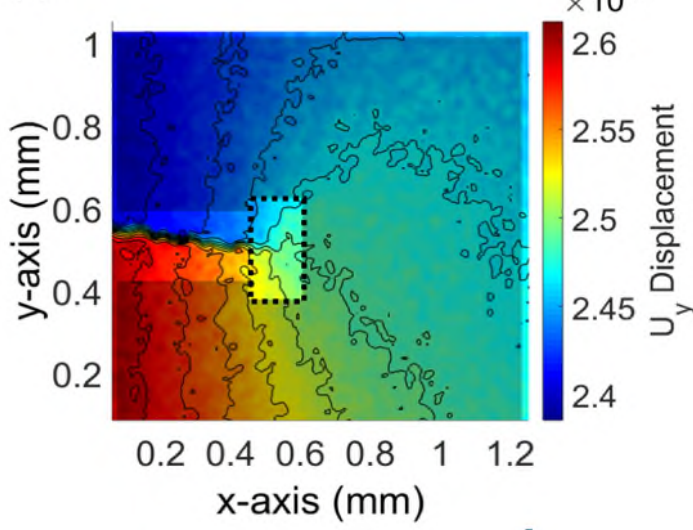

Figure 10: Examples of the selection of the initial J-integral contour (shown by black dotted box) and the excluded region ( $\sim 2 \mathrm{~mm}$ wide) along the crack for a) JMAN-S analysis of EDXD data (Von Mises stress map shown); b) JMAN analysis of DIC data (map of the displacement component perpendicular to crack plane shown).

The changes in the elastic stress intensity factor change experienced by the crack with the change in load within single cycles (i.e. at the same crack length for each map) are presented in Figure 11; data are presented from single cycles selected during phase 1, 2 and 3 of the experiment. The figure shows a good agreement between the stress intensity factor ranges obtained using EDXD, DIC and ASTM. The JMAN and JMAN-S analyses assume linear elastic properties, which are also assumed in the ASTM calculation. Since the fatigue loading in a single cycle is essentially elastic, good agreement should expected between the applied stress intensity factor range (ASTM) and that extracted from the DIC-measured displacement field. Indeed, the agreement of the DIC analysis with the ASTM value verifies that the ASTM equation can be applied (i.e. elastic loading) in this case. The agreement between the DIC and EDXD analyses also confirms that strain mapping by EDXD can be used to measure the change in the elastic field and then extract the cyclic stress intensity factor range. Non-linear cyclic plasticity at the crack tip, which is indicated by the small changes in the FWHM position (Fig 
9), would tend to reduce the elastic strain field that is measured directly by diffraction; this may be the cause of the tendency for the EDXD analysis to provide slightly lower values than the DIC and ASTM values at high applied loads.

This methodology therefore allows direct characterisation of the applied elastic strain energy field, without requiring any knowledge of the specimen geometry, crack length or applied loads. It may be used in future studies to investigate the effects of fatigue overloads, under loads and phenomena such as crack closure on the crack field that acts on the crack tip to control crack propagation.

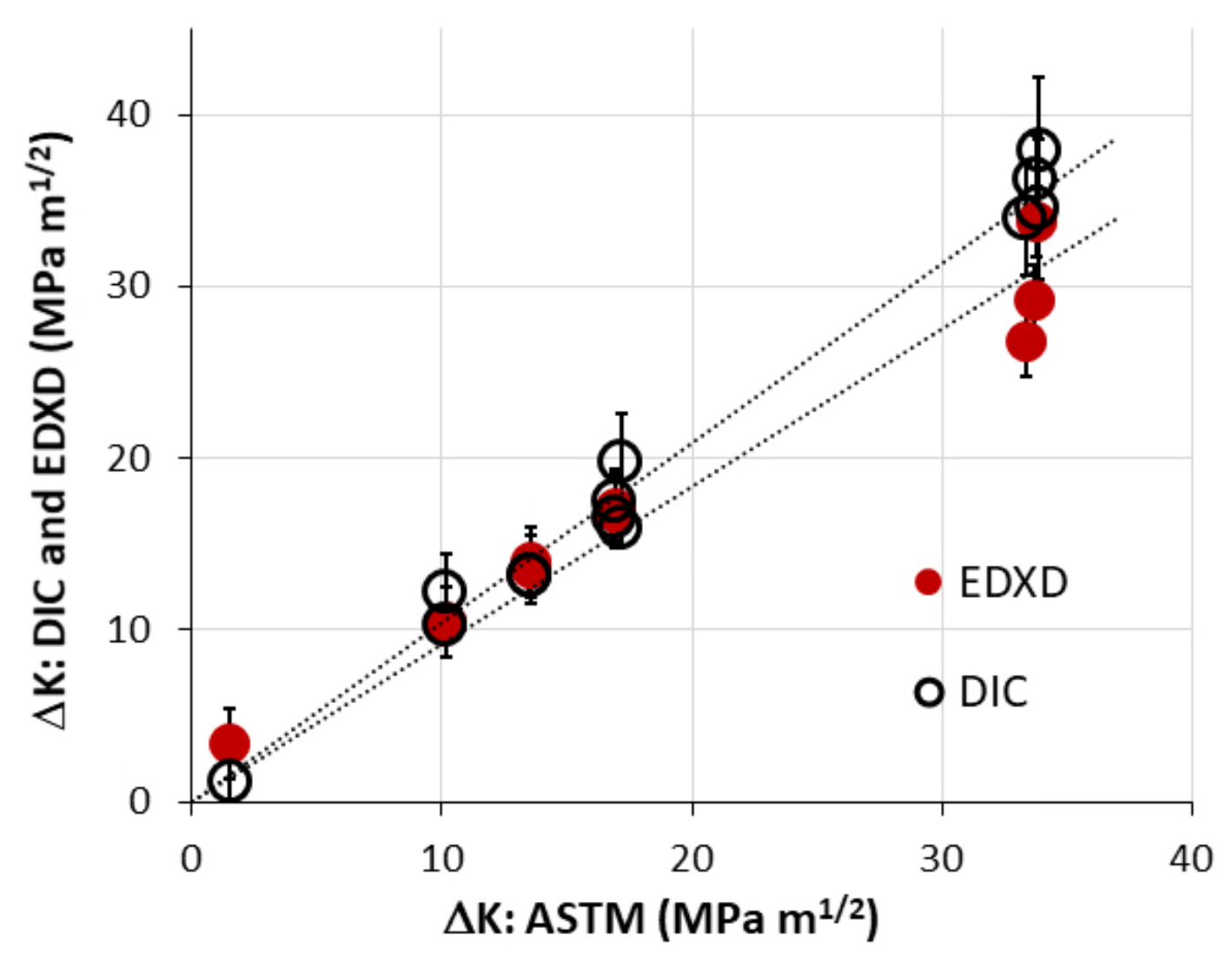

Figure 11: Stress intensity factor ranges obtained from DIC and EDXD.

\section{Conclusion}

Displacement and elastic strain maps, for a fatigue crack propagating in the HAZ of a welded Cr2Ni4MoV bainitic steel, have been obtained using Digital image correlation (DIC) and Energy Dispersive X-ray diffraction (EDXD), respectively. Movement of the crack tip was tracked via 
a phase congruency analysis of its opening displacement field and the EDXD data allowed tracking of the cyclic small-scale yielding zone. The data enabled an analysis of the cyclic crack field in a weld under controlled conditions of predominantly elastic deformation.

Both DIC and EDXD full field data provided inputs to finite element analyses (JMAN and JMANS) that could be used directly as boundary conditions to obtain an elastic stress intensity factor range, with no knowledge required of the specimen geometry, crack length or applied loads. Good agreement between the results obtained via EDXD and DIC data shows that synchrotron EDXD mapping could be used to reliably measure the crack tip elastic field in more complex situations that cannot be accessed by DIC; e.g. within a thick specimen and under the influence of residual stresses that may be introduced by overload cycles.

\section{Acknowledgements}

The EDXD observations were carried out at JEEP I12 beamline of the UK Diamond Light Source as experiment EE13579. The assistance of Dr T. Connolly (Diamond Light Source) with the experiment, $\operatorname{Dr}$ A. Cinar (Sheffield University) with the phase congruency method, $\operatorname{Dr}$ C. Simpson and Dr M. Mostafavi (University of Bristol) for their insights into XRD spectrum peak fitting are gratefully acknowledged.

\section{References}

[1] G. P. Cherepanov, "Crack propagation in continuous media," J. Appl. Math. Mech., vol. 31, no. 3, pp. 503-512, Jan. 1967.

[2] J. R. Rice, "A Path Independent Integral and the Approximate Analysis of Strain Concentration by Notches and Cracks," J. Appl. Mech., vol. 35, no. 2, p. 379, Jun. 1968.

[3] J. W. Hutchinson, "Singular behaviour at the end of a tensile crack in a hardening material," J. Mech. Phys. Solids, vol. 16, no. 1, pp. 13-31, Jan. 1968.

[4] J. R. Rice and G. F. Rosengren, "Plane strain deformation near a crack tip in a power- 
law hardening material," J. Mech. Phys. Solids, vol. 16, no. 1, pp. 1-12, Jan. 1968.

[5] X.-K. Zhu and J. A. Joyce, "Review of fracture toughness (G, K, J, CTOD, CTOA) testing and standardization," Eng. Fract. Mech., vol. 85, pp. 1-46, May 2012.

[6] J. A. Begley and J. D. Landes, "The J Integral as a Fracture Criterion," in Fracture Toughness: Part II, H. T. Corten, Ed. West Conshohocken, PA: ASTM International, 1972, pp. 1-23.

[7] Y. Y. Liu and F. S. Lin, "A mathematical equation relating low cycle fatigue data to fatigue crack propagation rates," Int. J. Fatigue, vol. 6, no. 1, pp. 31-36, Jan. 1984.

[8] Y. Lambert, P. Saillard, and C. Bathias, "Application of the J Concept to Fatigue Crack Growth in Large-Scale Yielding," in STP969-EB Fracture Mechanics: Nineteenth Symposium, T. Cruse, Ed. 100 Barr Harbor Drive, PO Box C700, West Conshohocken, PA 19428-2959: ASTM International, 1988, pp. 318-329.

[9] C. F. Shih, B. Moran, and T. Nakamura, "Energy release rate along a three-dimensional crack front in a thermally stressed body," Int. J. Fract., vol. 30, no. 2, pp. 79-102, 1986.

[10] H. E. Coules, G. C. M. Horne, K. Abburi Venkata, and T. Pirling, "The effects of residual stress on elastic-plastic fracture propagation and stability," Mater. Des., vol. 143, pp. 131-140, Apr. 2018.

[11] T. H. Becker, M. Mostafavi, R. B. Tait, and T. J. Marrow, "An approach to calculate the J-integral by digital image correlation displacement field measurement," Fatigue Fract. Eng. Mater. Struct., vol. 35, no. 10, pp. 971-984, Oct. 2012.

[12] D. M. Parks, "The virtual crack extension method for nonlinear material behavior," Comput. Methods Appl. Mech. Eng., vol. 12, no. 3, pp. 353-364, Dec. 1977.

[13] S. M. Barhli et al., "Obtaining the J-integral by diffraction-based crack-field strain mapping," Procedia Struct. Integr., vol. 2, pp. 2519-2526, Jan. 2016.

[14] S. M. Barhli et al., "Synchrotron X-ray characterization of crack strain fields in polygranular graphite," Carbon N. Y., vol. 124, pp. 357-371, Nov. 2017. 
[15] J. E. Huber, F. Hofmann, S. Barhli, T. J. Marrow, and C. Hildersley, “Observation of crack growth in a polycrystalline ferroelectric by synchrotron X-ray diffraction," Scr. Mater., vol. 140, pp. 23-26, Nov. 2017.

[16] T. Wigger et al., "In situ mapping of normal strains in the field of a growing fatigue crack in a steel weld using digital image correlation and energy dispersive synchrotron X-ray diffraction," Int. J. Fatigue, vol. 115, pp. 11-19, Oct. 2018.

[17] M. Drakopoulos et al., "I12: the Joint Engineering, Environment and Processing (JEEP) beamline at Diamond Light Source," J. Synchrotron Radiat., vol. 22, no. 3, pp. 828-838, May 2015.

[18] A. International, "ASTM E647-15e1, Standard Test Method for Measurement of Fatigue Crack Growth Rates," West Conshohocken, PA, 2015.

[19] F. Bachmann, R. Hielscher, and H. Schaeben, "Texture Analysis with MTEX - Free and Open Source Software Toolbox," Solid State Phenom., vol. 160, pp. 63-68, Feb. 2010.

[20] R. Hielscher and H. Schaeben, "A novel pole figure inversion method: specification of the MTEX algorithm," J. Appl. Crystallogr., vol. 41, no. 6, pp. 1024-1037, Dec. 2008.

[21] C. Simpson, "PyXe: XRD Strain Analysis," 2016.

[22] M. Mostafavi et al., "Dynamic contact strain measurement by time-resolved stroboscopic energy dispersive synchrotron X-ray diffraction," Strain, vol. 53, no. 2, p. e12221, Apr. 2017.

[23] V. K. Pecharsky and P. Y. Zavalij, "The Powder Diffraction Pattern," in Fundamentals of Powder Diffraction and Structural Characterization of Materials, 2nd Editio., Boston, MA: Springer US, 2009, pp. 151-202.

[24] B. Pan, K. Qian, H. Xie, and A. Asundi, "Two-dimensional digital image correlation for in-plane displacement and strain measurement: a review," Meas. Sci. Technol., vol. 20, no. 6, p. 62001, 2009.

[25] M. Guizar-Sicairos, S. T. Thurman, and J. R. Fienup, "Efficient subpixel image 
registration algorithms," Opt. Lett., vol. 33, no. 2, pp. 156-158, Jan. 2008.

[26] A. F. Cinar et al., "An autonomous surface discontinuity detection and quantification method by digital image correlation and phase congruency," Opt. Lasers Eng., vol. 96, pp. 94-106, Sep. 2017.

[27] Peter Kovesi, "Image Features from Phase Congruency," Videre J. Comput. Vis. Res., vol. 1, no. 3, pp. 1-26, 1999.

[28] T. Gnäupel-Herold, "ISODEC: software for calculating diffraction elastic constants," J. Appl. Crystallogr., vol. 45, no. 3, pp. 573-574, Jun. 2012.

[29] M. A. Weisser, A. D. Evans, S. Van Petegem, S. R. Holdsworth, and H. Van Swygenhoven, "In situ room temperature tensile deformation of a 1\% CrMoV bainitic steel using synchrotron and neutron diffraction," Acta Mater., vol. 59, no. 11, pp. 4448-4457, Jun. 2011.

[30] R. Hill, "The Elastic Behaviour of a Crystalline Aggregate," Proc. Phys. Soc. Sect. A, vol. 65, no. 5, pp. 349-354, 1952.

[31] W. Pantleon, "Resolving the geometrically necessary dislocation content by conventional electron backscattering diffraction," Scr. Mater., vol. 58, no. 11, pp. 994997, Jun. 2008. 